\title{
Tecnura
}

\section{Perspectiva para el uso del modelo P6 de atención en salud bajo un escenario soportado en IoT y blockchain}

\section{Perspective for the use of the p6 model of health under a scenario supported by the IoT and Blockchain}

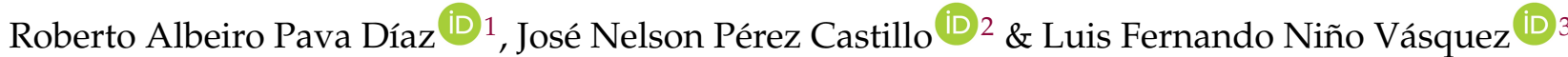

Fecha de Recepción: 11 de Junio de 2020

Fecha de Aceptación: 3 de noviembre de 2020

Cómo citar: Pava-Díaz., R.A. Pérez-Castillo., J.N. y Niño-Vásquez., L.F. (2021). Perspectiva para el uso del modelo P6 de atención en salud bajo un escenario soportado en IoT y blockchain. Tecnura, 25(67), 112-130. https:/ / doi.org/10.14483/22487638.16995

\section{Resumen}

Objetivo: Se introduce la tecnología blockchain, incluidas sus principales características. También se discute el modelo de medicina P6 para la atención centrada en el paciente, y se presentan las aplicaciones de la tecnología blockchain como una capa de seguridad e interoperabilidad para dispositivos médicos IoT y sistemas de información hospitalarios.

Metodología: Se realizó una revisión de las publicaciones registradas en las colecciones bibliográficas de IEEE Xplore y Scopus, con un filtro sobre las publicaciones enfocadas en blockchain, IoT y salud. El análisis de los artículos se enfocó en el planteamiento de un escenario de congruencia funcional de estos tres elementos.

Resultados: Se presentan las aplicaciones de blockchain e IoT para el cuidado integral de la salud, con una esquematización de la interacción de dicha tecnología entre los sistemas de información hospitalarios y los sensores médicos de IoT para la creación de las condiciones necesarias en la implementación de la medicina P6.

Conclusiones: Se identificó la aplicación de la tecnología blockchain como la capa de interoperabilidad necesaria entre sistemas de información hospitalarios, centros de investigación, pacientes, médicos y comunidad interesada; para generar un entorno confiable de flujo de información entre los diferentes actores que dinamice los procesos de investigación y atención en salud.

Palabras clave: blockchain, IoT, modelo de atención $\mathrm{P} 6$, cuidado de la salud, interoperabilidad.

\section{Abstract}

Objective: Blockchain technology, including its main features, is introduced. The P6 medicine model for

\footnotetext{
${ }^{1}$ Ingeniero de Sistemas, especialista en Redes de Alta Velocidad y Distribuidas, magíster en Ingeniería de Sistemas y Computación. Docente de la Universidad Distrital Francisco José de Caldas. Bogotá D. C., Colombia. Correo electrónico: rapavad@udistrital.edu.co

${ }^{2}$ Ingeniero de Sistemas, especialista Sistemas de Información Geográfica y Cartografía, magíster en Teleinformática y doctor en Informática. Docente de la Universidad Distrital Francisco José de Caldas. Bogotá D. C., Colombia. Correo electrónico: jnperezc@udistrital.edu.co

${ }^{3}$ Ingeniero de Sistemas, magíster en matemáticas, magíster en Ciencias de la Computación, doctor en Ciencias de la Computación. Docente de la Universidad Nacional de Colombia, sede Bogotá. Bogotá D. C., Colombia. Correo electrónico: lfninov@unal.edu.co
} 
patient-centered care is also shown, and finally, the applications of Blockchain technology as a security and interoperability layer for $I o T$ medical devices and hospital information systems is presented.

Methodology: A review of the publications registered in the bibliographic collections of IEEE Xplore and Scopus was carried out, with a filter on publications focused on Blockchain, IoT and health, in addition. The analysis of the articles focused on the approach of a functional congruence scenario of these three elements.

Results: Blockchain and IoT applications for comprehensive health care are presented, with an outline of the interaction of Blockchain technology between hospital information systems and IoT medical sensors to create the necessary conditions in the implementation of the P6 medicine.

Conclusions: The application of Blockchain technology was identified as the necessary interoperability layer to allow interoperability between hospital information systems, research centers, patients, doctors and the interested community, to generate a reliable environment for the flow of information among the actors that allow it, and will enhance streamline research and healthcare processes.

Keywords: blockchain, IoT, P6 medicine, Interoperability and health care domains.

\section{Tabla de Contenidos}

INTRODUCTION

METODOLOGÍA

\section{FUNDAMENTOS DE BLOCKCHAIN}

Aspectos generales en una blockchain . . . . 114

Antecedentes teóricos . . . . . . . . . . . 116

\section{CUIDADO DE LA SALUD}

\section{BLOCKCHAIN EN EL SECTOR SALUD}

Registros médicos electrónicos . . . . . 119

\section{BLOCKCHAIN, IoT Y SALUD}

Monitoreo remoto del paciente . . . . . . 122

Cadena de suministro farmacéutica . . . . . 122

Gestión de compras de suministros médicos 122

\section{CONCLUSIONES}

\section{REFERENCIAS}

\section{INTRODUCTION}

Actualmente la medicina se está orientando hacia el paciente, esto se refleja en el nuevo modelo $P 6$ : personalized, predictive, preventive, participatory, psycho cognitive, public, de atención integral para la salud, el cual requiere la generación de escenarios que permitan compartir y procesar información médica, de forma segura, interoperable y trazable. Por otro lado, blockchain ha sido catalogada como una de las tecnologías con mayor grado disruptivo en la última década; desde sus inicios, en 2008, ha transformado el sector financiero, económico y legal, pero más allá de las aplicaciones referentes a dinero digital, la tecnología de registro distribuido como blockchain ha generado interés tanto en el sector académico como del productivo, y se han diseñado soluciones para: registro y certificado de documentos, identificación de individuos, sistema de votación electrónica y registro de valor.

Por esto, blockchain junto con IoT se perfilan como el soporte tecnológico fundamental para un cuidado de la salud centrado en el paciente. A continuación se describe la metodología utilizada, seguido de una introducción a la tecnología blockchain y una contextualización del panorama general en la aplicación de blockchain e IoT en el sector salud. Por último, se presentan las conclusiones de la interacción IoT, blockchain para un modelo de medicina P6. 


\section{METODOLOGÍA}

Se realizó una consulta en las bases de datos de IEEE Xplore y Scopus, con la cadena de búsqueda (Blockchain AND (IoT OR 'P6 medicine' OR 'health care')) en el periodo entre 2009 y 2019. Posteriormente se realizó una clasificación manual de artículos, tomando como criterios título, abstract y conclusiones; el subconjunto seleccionado para lectura permitió extraer los elementos relativos a las características principales de blockchain y sus mecanismos de aplicación junto con IoT en salud. Finalmente, se agregó bibliografía secundaria relacionada en los documentos analizados.

\section{FUNDAMENTOS DE BLOCK- CHAIN}

Blockchain inicia en 2008 con la publicación informal de un documento técnico en un foro de criptografía, en el cual se describe un sistema de dinero electrónico denominado criptomoneda, basado en una red distribuida y descentralizada que da solución al problema del doble gasto; esta criptomoneda se acuñó como bitcoin (Nakamoto, 2008). Blockchain ha impactado nuestra sociedad al posibilitar la creación de escenarios que no requieren un entorno centralizado soportado en un tercero de confianza, quien debe garantizar la integridad y confidencialidad de la información (Ahram et al., 2017). En su inicio blockchain se utilizó principalmente en economía para la creación de criptomonedas (Brandon, 2020), pero su uso se extendió a entornos donde se requería información confiable entre un grupo de usuarios,lo que se refleja en soluciones propuestas para certificación de documentos, votaciones, cadena de suministro y logística, identificación, gobernabilidad y salud (Nomura Research Institute, 2016).

\section{Aspectos generales en una blockchain}

Blockchain se define como un registro de datos distribuido y descentralizado que preserva un conjunto de transacciones almacenadas en unidades denominadas bloques, los cuales se enlazan secuencialmente en el tiempo. Las transacciones son verificadas y aprobadas mediante la aplicación de un protocolo de consenso entre los miembros de la red antes de ser registradas en la blockchain, y una vez se ha agregado un bloque no podrá ser eliminado o modificado sin el consentimiento de la mayoría (Antonopoulos, 2014).

Las características de una blockchain son:

- Privacidad. El diseño de una blockchain permitirá un anonimato parcial o total en las transacciones. Así mismo, posibilita asignar permisos a los nodos de la red en tres configuraciones: a) pública: todos los participantes pueden leer, validar y agregar transacciones; b) consorcio: un subconjunto de participantes tiene el rol de validar y agregar transacciones, con una lectura de información que puede ser pública, y d) privada: dispone de un control de permisos centralizado para administrar los nodos. Por ejemplo, la red de bitcoin es una blockchain pública por lo cual se pueden adicionar nodos libremente y es posible realizar la trazabilidad completa de las transacciones, y en caso de conocer el propietario de una llave pública, se tendrá la asociación entre usuario y transacciones (Feng et al., 2019).

- Escalabilidad. Al ser un sistema distribuido y descentralizado, la escalabilidad de la red, medida en el número de transacciones por segundo (TPS), está limitada por elalgoritmo de consenso implementado en la blockchain. Por ejemplo, bitcoin soporta 7 TPS; ethereum, 15 TPS; litecoin, 56 TPS; bitcoin cash, 60 TPS, y ripple, 1500 TPS (Gervais et al., 2016).

La Tabla I muestra datos comparativos del desempeño de las blockchain de bitcoin y ethereum (versión PoW), determinados por el número de transacciones, la cantidad de energía eléctrica consumida y el costo de confirmar una transacción. Estas dos blockchain 
Tabla I. Datos de funcionamiento de las blockchain de bitcoin y ethereum

\begin{tabular}{|l|c|c|c|c|c|c|c|}
\hline & \multirow{2}{*}{ TPS } & TWh & País de & KWh & \multicolumn{3}{|c|}{ Costo (USD) } \\
\cline { 5 - 9 } & & (año) & referencia & (trans.) & Mín. & Máx. & Actual \\
\hline Bitcoin & 7 & 48,74 & Singapur & 394 & 0 & 147 & 19 \\
\hline Ethereum & 15 & 8,93 & Etiopia & 47 & 0,0566 & 5,528 & 0,0908 \\
\hline
\end{tabular}

Fuente: elaboración propia.

Tabla II. Listado de Algoritmos de Consenso aplicados en Blockchain

\begin{tabular}{|c|c|}
\hline Algoritmo de consenso & Casos de aplicación \\
\hline Proof of work (PoW) & $\begin{array}{l}\text { Nakamoto (2008); Ethereum Foundation (2015); } \\
\text { Litecoin Foundation (2017); Bitcoincash (2017). }\end{array}$ \\
\hline Proof of stake (PoS) & $\begin{array}{l}\text { PIVX Community (2018); Bitmoney Inc. (2019); } \\
\text { Vasin (2014). }\end{array}$ \\
\hline Delegate proof of stake (DPoS) & $\begin{array}{l}\text { BitShares Blockchain Foundation (2013); } \\
\text { Cardano Foundation (2020); Trew, Brandon } \\
\text { y Dorier (2017); Block.one (2017). }\end{array}$ \\
\hline Leased proof of stake (LPoS) & Waves Technologies (2016); Nix Platform (2018). \\
\hline Proof of authority (PoA) & Kovan Github (2014). \\
\hline Proof of existence (PoE) & Silent Notary (2018); po.et Foundation (2018). \\
\hline Byzantine fault tolerance (BFT) & $\begin{array}{l}\text { The Linux Foundation (2017); Sajana, Sindhu y } \\
\text { Sethumadhavan (2018). }\end{array}$ \\
\hline Delegated byzantine fault tolerance (dPBFT) & Neo Foundation (2017). \\
\hline Practical byzantine fault tolerance (pBFT) & Seeley (2018). \\
\hline Proof of importance (PoI) & The NEM Group Ltd. (2018). \\
\hline Proof of brain (PoB) & Steem Inc. (2017). \\
\hline Proof of elapsed time (PoET) & Bowman y Gendal (2018). \\
\hline Proof of burn & $\begin{array}{l}\text { P4Titan (2014); Counterparty Inc. (2019); } \\
\text { Snow et al.(2018). }\end{array}$ \\
\hline Proof of space (PoSpace) & Burstcoin Inc. (2014); Alwen et al. (2015). \\
\hline Proofs of space and time (PoST) & Cohen (2019). \\
\hline Direct acyclic graphs (DAG) & Popov (2018). \\
\hline Ripple protocol consensus algorithm & Schwartz, Youngs y Britto (2018). \\
\hline
\end{tabular}

Fuente: elaboración propia. 
han sido las de mayor interacción en el mercado de criptomonedas y han presentado problemas de escalabilidad, con los consecuentes niveles de consumo de energía eléctrica similares a los de una nación como Singapur o Etiopia, situación que se traduce en elevadas tasas de comisión por transacción. Bitcoin inició sin costo de comisión por transacción y llegó a un máximo histórico de 147 USD el 24 de diciembre de 2017, actualmente se encuentra en 19 USD. La red de ethereum alcanzó el valor máximo por transacción el 2 de julio de 2018 (Brandon, 2020).

- Gobernanza. La transición de entornos centralizados a ambientes descentralizados -por ejemplo, el cambio de un sistema financiero controlado por los bancos a un sistema financiero autogestionado por la comunidad- elimina los riesgos generados por un control y presión que se puedan ejercer de forma directa sobre el sistema, pero demanda la modificación de políticas de gestión en el interior de las organizaciones,y la adaptación de marcos metodológicos de gestión corporativa, que permitan la correcta selección del tipo de blockchain conforme a las características de la organización (Vauplane, 2017, Hassan y Primavera, 2017).

- Sistema de consenso. El algoritmo de consenso establece el mecanismo por el cual se propende por garantizar la integridad de la información almacenada en la blockchain. Permite a los nodos de la red validar las transacciones y conservar un historial único con el objeto de prevenir un cambio fraudulento en la cadena de bloques (Bach, Mihaljevic y Zagar, 2018, Gramoli, 2020, Sankar, Sindhu y Sethumadhavan, 2017, Zheng et al., 2017). La Tabla II contiene una relación de los algoritmos de consenso propuestos para blockchain, con un conjunto de casos de aplicación para cada uno de ellos.

\section{Antecedentes teóricos}

La figura 1 es una síntesis de los antecedentes teóricos de blockchain. Inician con las funciones hash y su aplicación para firmas digitales, en los años 1976 y 1978, respectivamente. En 1991, se publica el concepto de cadena de marcas de tiempo para certificar la creación de un documento digital; esta hacía uso de la estructura de datos árbol de Merkle propuesta en 1980. En 1993, se presenta la prueba de trabajo y se implementa en hashcash como mecanismo para reducir el correo spam; pero es hasta la aparición de $b$ - money que se asientan los referentes para la implementación de un sistema de transferencia de valor distribuido y descentralizado. En 2009, aparece la primera generación de blockchain, desarrollada bajo los lineamientos propuestos por (Nakamoto, 2008) en bitcoin, y se centra en la creación de sistemas públicos, descentralizados y distribuidos para un registro inmutable de transacciones en procesos de transferencia de valor. Debido a las limitaciones de los lenguajes script se evoluciona a la generación 2.0, la cual es liderada por Vitalin Buterik, cofundador del proyecto ethereum. En esta generación se incluyen lenguajes Turingcompletos para potenciar el desarrollo de aplicaciones descentralizadas basadas en el uso de contratos inteligentes.

Por último, la generación 3.0 se orientó en los problemas encontrados en sus predecesores, principalmente en los algoritmos de consenso que determinan el número de transacciones por segundo, limitan la escalabilidad de la red y dificultan la interoperabilidad entre cadenas de bloques.

\section{CUIDADO DE LA SALUD}

La Organización Mundial de la Salud (WHO, por su sigla en inglés) define la salud como un estado de bienestar dado en tres dimensiones: física, mental y social. Igualmente, la concibe como un derecho fundamental de los individuos, $\mathrm{y}$ un requisito indispensable para una sociedad segura y en paz. La responsabilidad de la salud 
concierne a todos los pueblos del mundo, y requiere de un trabajo mancomunado que permita la transferencia de conocimientos y buenas prácticas para la toma de decisiones referentes a salud pública (World Health Organization, 2005). Esta concepción de salud está generando una transformación del modelo de atención, pasando de un esquema centrado en el médico (modelo P0) a un entorno orientado al paciente (modelo P6), el cual se encuentra basado en seis características: un sistema de salud con información pública e interoperable entre los diferentes actores, que permita una mayor participación del paciente en su tratamiento y con facilidades de apoyo psicocognitivo de la comunidad, enfocando el sistema de salud en una medicina preventiva, predictiva y personalizada.

En la figura 2 se representa el desarrollo incremental de los modelos de atención en salud, partiendo desde P0 hasta P6 (Bragazzi, 2013, Bragazzi y Del Puente, 2013). En el modelo P0, el médico era el actor principal y el paciente participaba de forma pasiva en su proceso de recuperación; en contraste, el modelo P6 tiende por una medicina de precisión, en la cual la sanación se personaliza al paciente, quien ahora tiene un papel activo que le permite integrar su núcleo familiar y personal, además del apoyo de la comunidad interesada por un padecimiento particular.

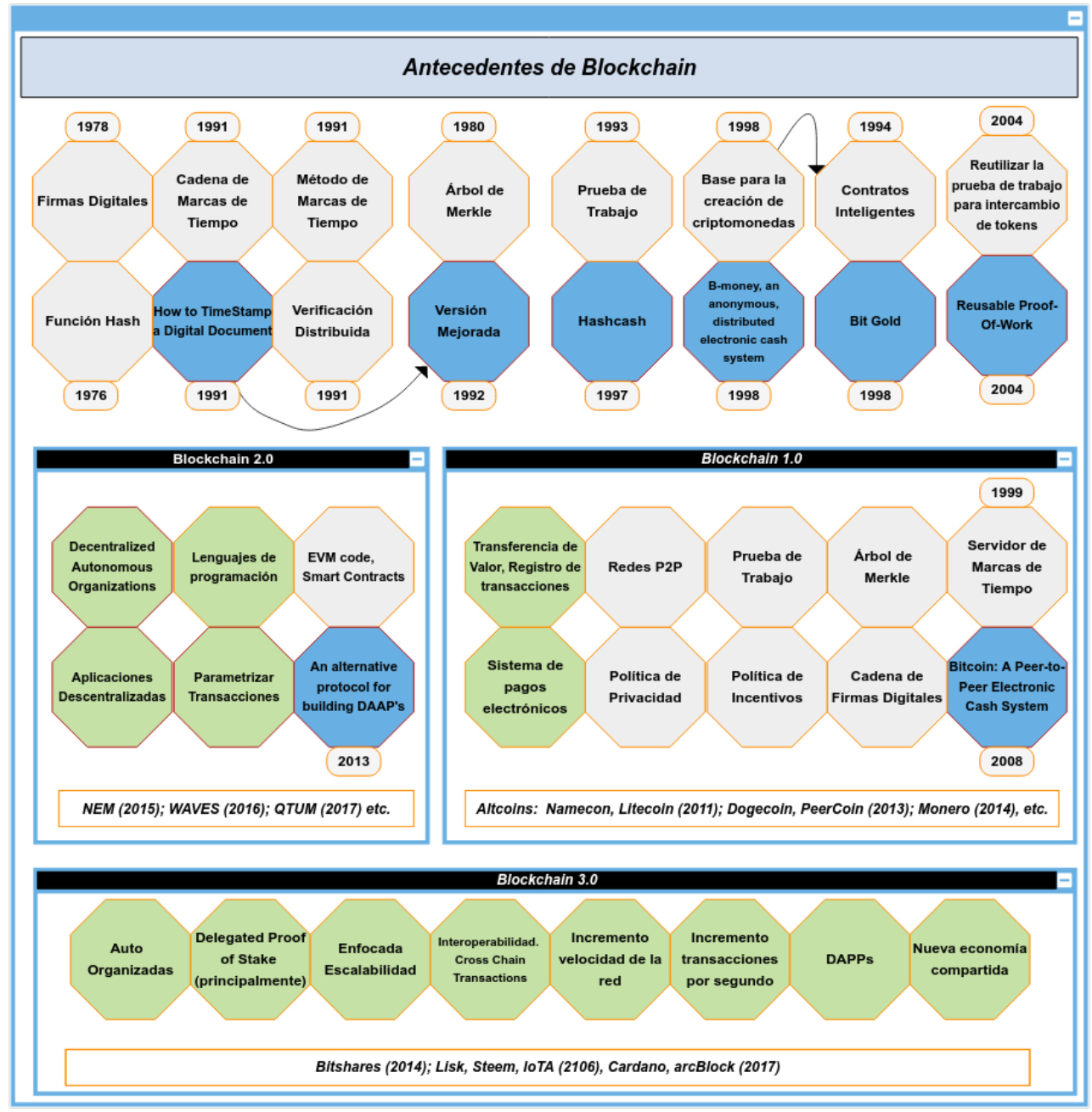

Figura 1. Antecedentes teóricos y generaciones de la tecnología blockchain 


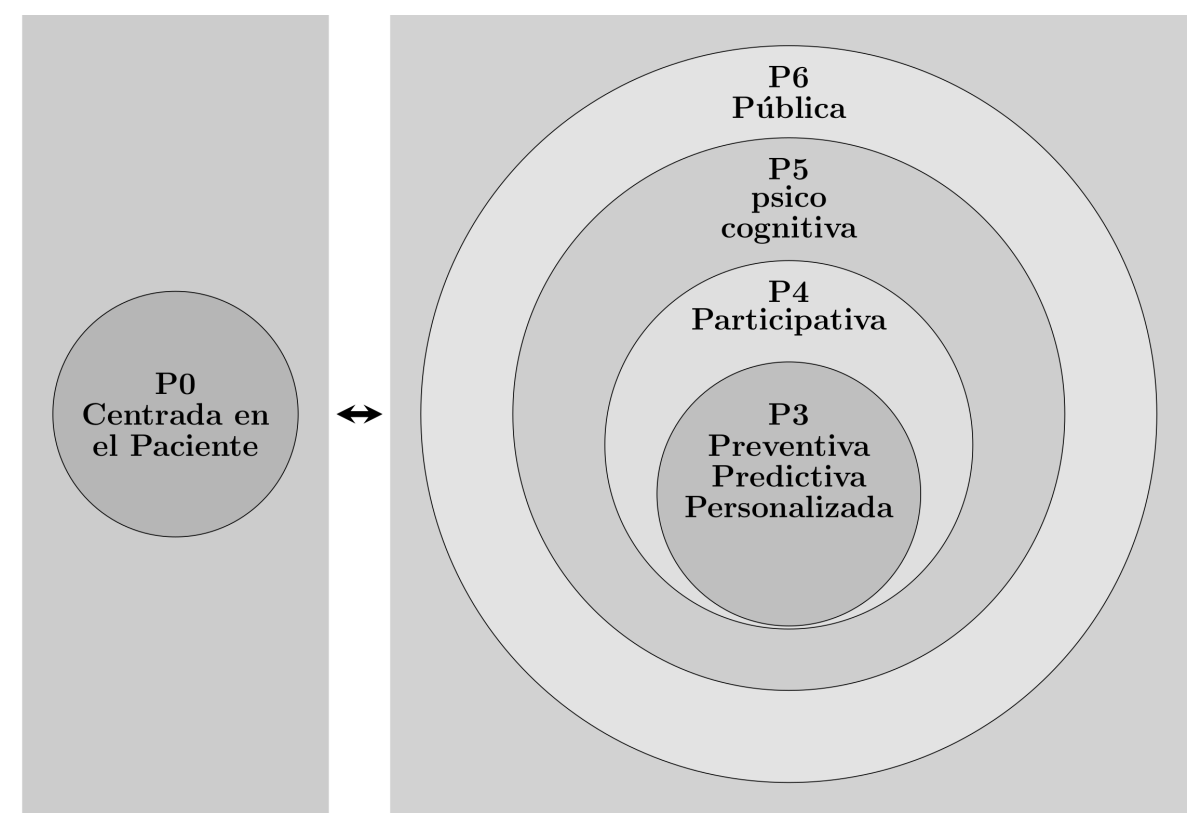

Figura 2. Evolución del modelo de atención en salud

Fuente: elaboración propia.

La investigación actual se conduce hacia la implementación del modelo P6, lo cual implica cambios en la gestión del cuidado de la salud con un nuevo comportamiento de los actores involucrados. En este contexto blockchain e IoT emergen como soporte tecnológico que posibilitan la prestación del servicio orientado al paciente (Hölbl, Kompara, Kamišali y Nemec, 2018), con una asertiva auditoría de los eventos de atención en salud y prescripción de medicamentos. Esto facilita la ubicuidad de la información médica, con recolección de datos médicos del paciente de forma automática y consensuada, mediante el despliegue de mecanismos de autorización para la visualización de datos clínicos de forma controlada, lo cual permite compartir de forma anónima su información clínica en procesos de diagnóstico e investigación médica.

En este contexto, la figura 3 ilustra la interacción de blockchain, IoT y el modelo P6 de atención en salud. Como se mencionó anteriormente, la salud se percibe como un estado mediado por un bienestar mental, físico, y social de la persona, razón por la cual la atención en salud debe incluir estos tres factores enfocados desde la perspectiva del paciente, por ser quien mejor conoce su estado, lo cual permitirá un mejor diagnóstico y tratamiento por parte de los profesionales de la salud.

El modelo P6 requiere un flujo confiable de información médica sensible entre pacientes, prestadores de salud, instituciones de investigación, comunidades de interés y núcleo familiar. Blockchain permitirá el diseño de un escenario seguro, confiable, auditable y ubicuo para compartir información médica; facilitará, por ende, los procesos de investigación clínica para diagnóstico oportuno y personalizado del paciente; agregará una capa de seguridad para la administración de los dispositivos médicos IoT que monitorean el estado de salud físico del paciente.

Finalmente, un ambiente de flujo de información con estas características provee las condiciones necesarias para la atención integral en salud que facultará una medicina predictiva con la capacidad de predecir ocurrencias de enfermedades por la proyección de estados futuros de salud del paciente, con lo cual se logra una medicina preventiva y personalizada dotada de herramientas para diaǵnos- 


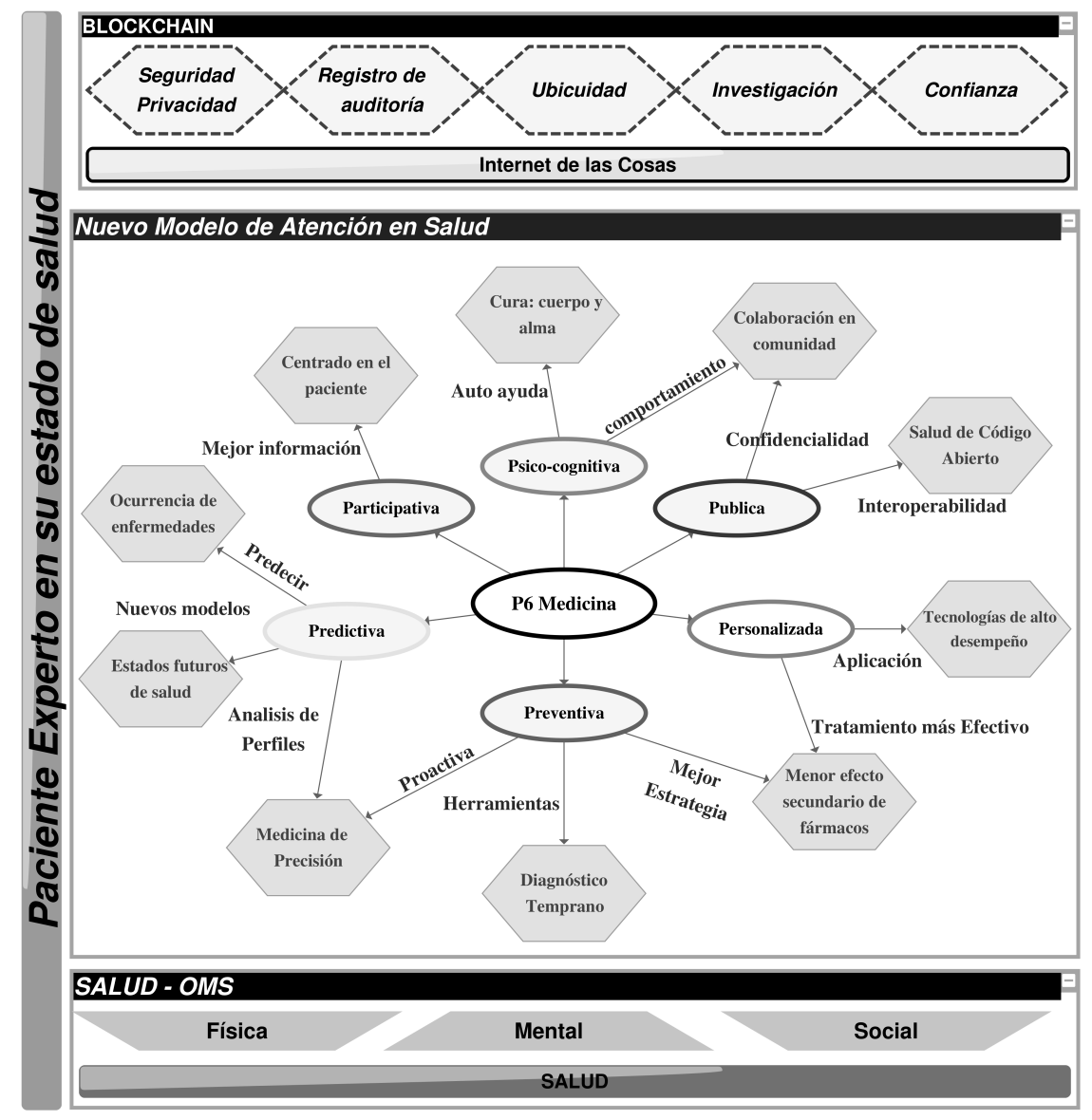

Figura 3. Interacción de Blockchain con el modelo p6 de atención en salud Fuente: elaboración propia.

tico temprano con tratamientos personalizados de mayor eficacia y mitigación de posibles efectos adversos.

Para finalizar, la información generada en los procesos de atención médica debe ser pública, previo consentimiento de los involucrados, que habilite la colaboración de las comunidades en entornos confidenciales, con una participación activa del paciente que le permita acceder a un apoyo psicosocial en su proceso de enfermedad.

\section{BLOCKCHAIN EN EL SECTOR SALUD}

Blockchain se ha aplicado principalmente en dos áreas: a) registro electrónico de historial clínico con la definición de métodos para consultar y compartir información, y b) verificación sobre la cadena logística relacionada con la industria farmacéutica (Abujamra y Randall, 2019, Kuo, Kim y Ohno-machado, 2017, McGhin, Choo, Liu y He, 2019).

\section{Registros médicos electrónicos}

Los actuales sistemas de gestión de información médica (electronic health records -EHR-) no serán sustituidos por sistemas tipo blockchain; por el contrario, se espera que estos se adapten y coexistan para desplegar las ventajas que ofrece la tecnología de registro distribuido (Pirtle, 2018) y el cumplimiento de normas de seguridad de la información (Arévalo-Ascanio, Bayona-Trillos y 
Rico-Bautista, 2015). A continuación, se describen las características objetivo que modificará una implementación de Blockchain en un sistema de gestión de registros médicos electrónicos (Mettler, 2016).

- Acceso seguro a los datos. Se agrega una capa de seguridad que permite una identidad anónima de los usuarios, con procesos de identificación y autorización de pacientes legítimos validados en la blockchain. Esta identidad anónima facilita la colaboración de los pacientes con otros individuos $u$ organizaciones, la expedición de seguros médicos en un entorno confiable y la corroboración de resultados de ensayos clínicos e investigaciones (Dubovitskaya et al., 2018).

- Intercambio de información médica (health information exchange -HIE-). El acceso e integración del historial médico que se almacena en las bases de datos centralizadas posibilita la construcción de un historial clínico único del paciente, lo que facilita la interacción entre médicos, especialistas, terapeutas y farmacias; independientementedel sitio donde se realice la atención. Este escenario requiere la implementación de mecanismos de interoperabilidad entre blockchain $\mathrm{y}$ prestadores de salud (Peterson, Deeduvanu, Kanjamala y Boles, 2016).

- Gestión gubernamental de registros médicos (e-Health records). El caso más representativo se encuentra en Estonia (Williams-Grut, 2016), donde el sistema de salud se ha soportado en blockchain, y su implementación se ha realizado en conjunto con la compañía Guardtime (2019). En esta blockchain interactúan los ciudadanos, los prestadores de salud y las empresas aseguradoras del sector, y se lleva un registro de consultas y actualizaciones realizadas sobre los registros médicos (Griggs et al., 2018).
- Investigación médica. A continuación se presentan las cuatro aplicaciones/escenarios más sobresalientes en la literatura diseñadas sobre blockchain, orientadas a procesos de investigación en medicina: a) diagnóstico colaborativo: en un entorno con acceso a la información descentralizada, los especialistas médicos pueden trabajar conjuntamente para generar un consenso sobre el estado de salud de un paciente basado en la evidencia médica registrada en su historia clínica, la información de la literatura científica y de las bases de datos biológicas (Talukder, Chaitanya, Arnold y Sakurai, 2018). b) Healthbank: sistema de intercambio de información bajo el estándar de seguridad suizo (Swiss security standard), que permite compartir datos con médicos, centros de atención, familiares o público en general; y realizar transacciones sobre el historial clínico de un paciente con el objeto de conformar una red de investigación en medicina. El diseño del sistema permite la lectura de datos de múltiples orígenes y formatos médicos (HealthBank Cooperative, 2019). c) GemHealth Network: sistema de historial clínico implementado sobre ethereum (GemOS Inc, 2018) para la consulta de información médica actualizada por parte de un conjunto especialistas. Este tipo de sistemas son un factor clave para reducir problemas de negligencia médica por información incompleta, puesto que pondrá a disposición del personal médico todos los datos relacionados con episodios de salud del paciente (Mettler, 2016). d) MedRec: diseñado para realizar una integración con los sistemas de información desplegados en cada prestador de salud, con el objetivo de interconectarlos para la investigación médica (Ekblaw, Azaria, Vieira y Lippman, 2016, Nchinda, Cameron, Retzepi y Lippman, 2019).

El diseño usa una blockchain como capa intermedia que permite el registro y validación de los usuarios identificados como pacientes, médicos 
y centros de atención en salud; además de permitir la lectura de los repositorios médicos alojados en cada institución. Se implementó tomando como base el código fuente de ethereum (Ethereum Foundation, 2015), con la modificación en el cálculo de la llave pública para hacer uso de una cadena de texto con información de identificación del paciente e incluir el número de seguridad social o número único de identificación personal; y la adición de un servicio de traducción, similar a un DNS, entre la llave pública de un usuario y su respectiva dirección ethereum.

El acceso y flujo de la información se controla mediante tres contratos inteligentes: contrato 1, de registro de identidad (registrar contract $-\mathrm{RC}-$ ) responsable del registro único de usuarios, con la asociación entre la cadena usada para la identificación y su dirección ethereum. Contrato 2, de interacción paciente-proveedor (patient provider relationship contract -PPR-) donde se configura el mecanismo de comunicaciónentre un proveedor, quién almacena y administra los registros médicos, y un paciente que es el propietario de sus registros. El PPR crea un inventario de la información del proveedor y lo almacena en un listado de consultas tipo SQL para su persistencia en una base de datos relacional, junto con los datos necesarios para la conexión. Cada resultado generado por una consulta es certificado por el cálculo de su hash. Finalmente, este contrato implementa la asignación de permisos y el contexto para para que un paciente pueda compartir su historial médico. El contrato 3, de historial médico (summary contract-SSC-)recopila el listado de orígenes de datos registrado en los PPR para un usuario, y le permite al a un usuario el retiro o reingreso al sistema, característica fundamental para el cumplimiento de las normas referentes al derecho al olvido, conforme a la jurisprudencia de cada nación.

Por último, el sistema presenta cuatro módulos, la librería Backend, un cliente ethereum, el controlador de la base de datos y el administrador para el sistema de registros médicos electrónicos. La librería Backend es el conjunto de librerías que abstrae la comunicación con la blockchain e interactúa con el cliente ethereum. El cliente ethereum es implementación de un nodo completo, con la adición de un servicio de localización para la ejecución de los contratos SC, y para la notificación de las actualizaciones en las bases de datos de terceros, aplica un consenso mediado por una prueba de trabajo y propone como incentivo a los mineros el acceso a datos médicos anónimos. En el prototipo implementado se agregó una consulta de recompensa al contrato PPR, por ejemplo, un minero podría recibir los datos de los niveles de hierro de todos los pacientes en periodo de tiempo. Una vez un proceso de minado es finalizado, se actualiza el PPR y los usuarios lo confirman o rechazan ensus SSC. El controlador de la base de datos: proporciona una interfaz de comunicación con los repositorios de información locales de los prestadores de salud, conforme a los permisos establecidos en la blockchain. Y finalmente el administrador para el sistema de registros médicos electrónicos, responsable de administrar el historial médico almacenada por cada proveedor. El historial médico del paciente se encuentra distribuido entre todos sus prestadores de salud. El éxito de la interoperabilidad depende de la aplicación de estándares abiertos para el intercambio de información en salud, como por ejemplo el uso fast healthcare interoperability resources (Health Level Seven International, 2011).

\section{BLOCKCHAIN, IoT Y SALUD}

El uso de dispositivos para el monitoreo de pacientes (Medical IoT, MIoT) se ha incrementado en los últimos años, con un pronóstico de ventas de USD 538 millones para el periodo 2018-2022 (Infiniti Research Limited, 2018), además de un incremento en su aplicación en la cadena de fabricación y suministro de alimentos (Nivia y Jaramillo, 2018, Ruge y Pérez, 2017). Estos sensores generan información médica sensible del paciente y se configuran dentro de una red inalámbrica de alcance corporal (wireless body area networks -WBAN-). Estos sensores demandan de medios de transmisión confiables y seguros para preservar los datos clínicos 
del paciente (protected health information -PHI-), y facilitar su administración y su registro en el historial médico alojado en el prestador de salud (Crosby, Ghosh, Murimi y Chin, 2012).

\section{Monitoreo remoto del paciente}

Alblooshi, Salah y Alhammadi (2018) proponen un sistema de registro distribuido tipo ethereum, orientado a fabricantes de dispositivos médicos IoT (MIoT) pacientes y hospitales, para la administración de MIoT mediante el uso de contratos inteligentes. En esta blockchain se puede certificar la procedencia de un MIoT para mitigar el contrabando y la falsificación de dispositivos o auditar el derecho a la propiedad de estos. Los contratos inteligentes verifican la información de los MIoT, generando alertas de adulteración, desconexión o falsificación a los involucrados.

\section{Cadena de suministro farmacéutica}

La vigilancia en la fabricación y distribución de medicamentos es esencial para la creación y aplicación de políticas de salud pública gubernamental. El delito de falsificación de medicamentos es una industria multimillonaria con ganancias de USD 75000 millones, únicamente en Estados Unidos. Afecta principalmente a los países en vía de desarrollo, y se estima que un $30 \%$ de los medicamentos distribuidos en las farmacias han sido adulterados. Esta situación genera problemas como: afectación en el estado de salud del paciente, incluso ocasionar la muerte; pérdida de la confianza de la población en un medicamento o el laboratorio que lo produce; aumento del riesgo de generar resistencia a medicamentos antimicrobianos con la subsiguiente aparición de infecciones farmacorresistentes (World Health Organization, 2010, World Health Organization, 2018).

Blockchain ofrece un mecanismo para que los interesados en comercializar medicamentos puedan realizar una trazabilidad completa sobre un producto médico, obteniendo de forma confiable la información de producción, efectos secundarios reportados y la supervisión de las condiciones ambientales necesarias para su traslado y almacenamiento.

Finalmente, Modum.io AG (2017) se enfoca en implementar un sistema que permita el monitoreo confiable de las variables asociadas a dispositivos IoT durante el transporte de medicamentos, específicamente para un sensor de temperatura en su fase inicial (Modum.io AG, 2017, Bocek, Rodrigues, Strasser y Stiller, 2017). Modum.io AG está compuesto por:

- Un sensor de temperatura que opera en el rango $\left[-40^{\circ} \mathrm{C}, 70^{\circ} \mathrm{C}\right]$ con intervalos de lectura de un segundo hasta una semana. El sensor envía la información a un dispositivo móvil por BLE (bluetooth low energy) previamente conectado a través de NFC (near field communication).

- Una base de datos relacional en PostgreSQL que almacena los registros generados por sensores de temperatura y la información de los usuarios registrados en el sistema. Un servidor web permite la comunicación (vía RestAPI/JSON) con la aplicación móvil y la blockchain.

- La blockchain implementa un nodo completo ethereum, en el cual se lleva un registro inmutable de la temperatura; y ejecuta un contrato inteligente que invalida el producto cuando se detecte un valor fuera del rango permitido, lo cual genera un reporte que se envía al distribuidor o fabricante; o valida la entrega del medicamento, aprobando las condiciones de transporte. Para el sistema de consenso se utiliza un algoritmo de prueba de existencia (Sankar, Sindhu y Sethumadhavan, 2017).

\section{Gestión de compras de suministros médicos}

Para los prestadores de salud se diseñan procesos de adquisición de suministros médicos orientados 
a mitigar los riesgos por material en condiciones no óptimas para su aplicación o que han sido falsificados. Celiz, De la Cruz y Sánchez (2018) proponen un modelo de gestión de compras basado en cuatro capas:

- Datos, responsable de la lectura de la información almacenada por los involucrados (vendedor, transportador, comprador) con definición de mecanismos para extracción, carga y transformación.

- IoT, conformada por una red de sensores implementada con Arduino UNO R3 board para medir la temperatura, humedad y ubicación del producto; los datos se transmiten a un servicio en la nube por medio de MQTT (message queue telemetry transport) y se almacena bajo un modelo no relacional en Dynamodb.

- Blockchain es el registro inmutable de los datos generados por los sensores y supervisión de las condiciones de los productos mediante contratos inteligentes. Se implementó en Hyperledger sobre Amazon Cloud y se desplegó en Hyperledger Fabric (The Linux Foundation, 2017).

- La capa de presentación, desplegada sobre una aplicación web que permite la administración del sistema, crear usuarios, órdenes de compra, y realizar seguimiento en tiempo real.

Para finalizar, en la figura 4 se ilustra la aplicación de blockchain en la creación de entornos confiables para uso de sensores médicos e integración entre sistemas de información hospitalarios para generar un proceso de atención en salud bajo el modelo P6 (Zhang, Xue y Huang, 2016). Blockchain se proyecta como la solución para la creación de una red descentralizada para IoT, con gestión de seguridad, confidencialidad y políticas de privacidad, la cual presenta dispositivos
IoT heterogéneos, con características variables en capacidad de procesamiento, almacenamiento y batería, que pueden presentar problemas relacionados con: confidencialidad, integridad, disponibilidad, autentificación, privacidad ylectura en tiempo real (Sedrati, Abdelraheem y Raza, 2018, Kshetri, 2017, Shrobe, Shrier y Pentland, 2018).

Los dispositivos MIoT se pueden autorizar mediante blockchain con el objetivo de mitigar riesgos de adulteración (firmware modificado) del dispositivo o acceso no autorizado a la información de este; además, esta autorización permitirá realizar auditorías, ya que se dispone de un registro inmutable de acciones realizadas sobre los MIoT. Por otro lado, los sensores IoT junto con la tecnología de registro distribuido están tomando relevancia en la cadena de distribución en general, y en particular en la farmacéutica, por su utilidad para mitigar la distribución de medicamentos falsificados y llevar un control verificable y confiable de las condiciones de transporte y almacenamiento de los productos médicos, con la posibilidad de almacenar información adicional en sistemas relacionales tradicionales como PostgreSQL, tal como lo plantea el sistema Modum.io AG (Modum.io AG, 2017); con esto se obtiene un conjunto de información de carácter público, con acceso seguro e identidad anónima que permite la colaboración de individuos e instituciones para la atención en salud. Esta colaboración permitirá a la comunidad validar la información de reportes médicos además de activar una participación anónima y recompensada en ensayos clínicos enfocados en una medicina preventiva, personalizada y predictiva.

Por último, la integración de información de atención en salud junto con los mecanismos para compartirla de forma segura y anónima generan redes de interacción médica entre pacientes que les permiten obtener un apoyo psicocognitivo de la comunidad afectada por un padecimiento (Shae, 2017), un ejemplo de esta situación la tenemos en Gem Health Network (GemOS Inc, 2018). 


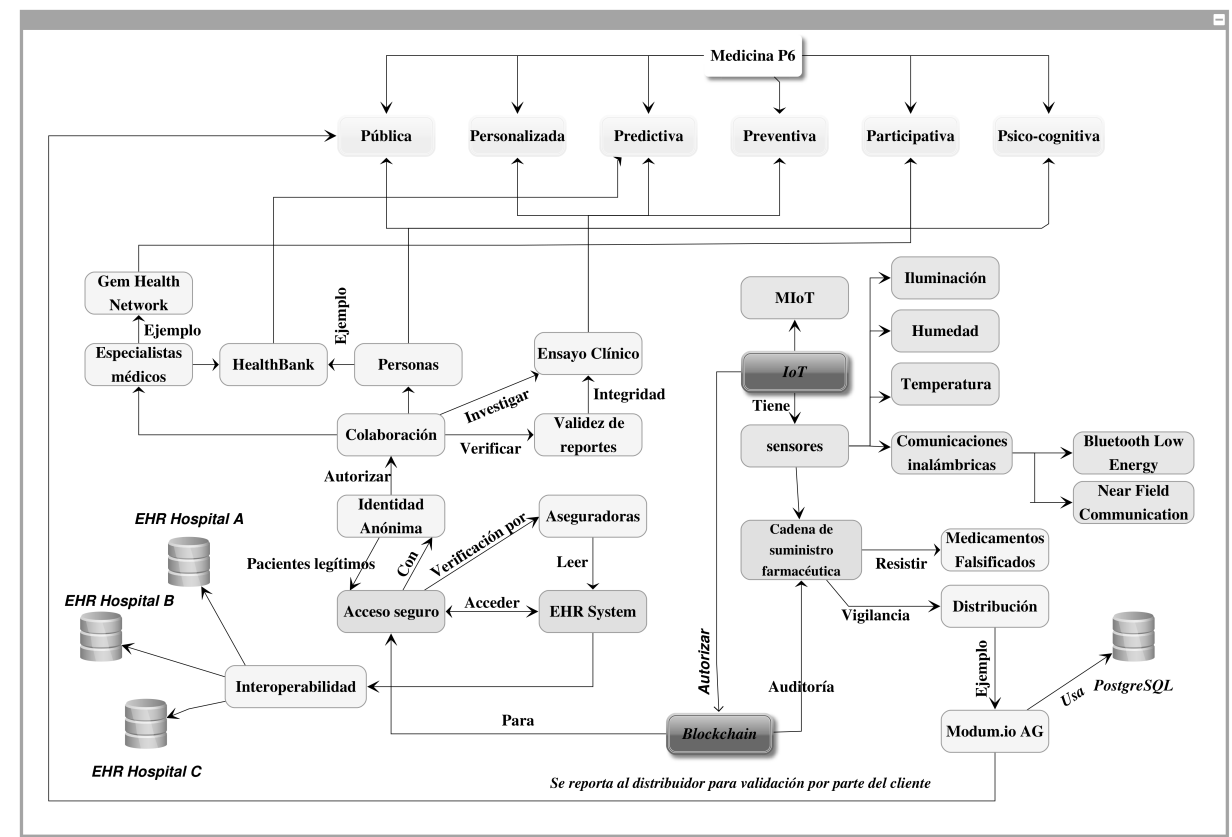

Figura 4. Aplicación de Blockchain e IoT en el sector de la salud

Fuente: elaboración propia.

\section{CONCLUSIONES}

El conjunto de escenarios de aplicación de blockchain, relacionados con la administración de datos clínicos y la gestión de dispositivos médicos IoT (MIoT), evidencia la proyección de esta tecnología para la generación de un modelo de atención en salud P6, puesto que facilita la gestión de datos clínicos descentralizados del paciente, y que actualmente se encuentran bajo el control de los prestadores de salud.

Las características de blockchain permitirán al paciente ejercer un control directo sobre su historia clínica, lo que provocará cambios en el modelo de operación de los prestadores de salud, en el papel que desempeña el paciente frente a su condición de salud y en el usufructo de la información clínica asociado a procesos de investigación médica. En este escenario, el paciente podrá decidir con quien desea compartir sus datos médicos y las condiciones para hacerlo; igualmente podrá apelar a su derecho al olvido, a eliminar o a requerir la anonimidad de sus datos médicos.
Los prestadores de salud deberán ajustar su plataforma tecnológica para permitir un entorno interoperable en el sector, que facilite el intercambio seguro de información entre pacientes, especialistas médicos, aseguradoras, farmacéuticas, centros de investigación y comunidad en general. Los contratos inteligentes ayudarán a automatizar tareas de verificación de condiciones; por ejemplo, la expedición de una póliza médica se podrá realizar con información real del estado de salud del paciente, y facilitará la resolución de dudas frente a coberturas o inhabilidades; por otro lado, el mercado de suministros médicos obtendrá un mecanismo para validar la calidad y autenticidad de un producto, lo que ayudaría a disminuir el riesgo de adquirir elementos adulterados, falsificados o de dudosa calidad debido a condiciones deficientes de almacenamiento y transporte.

\section{REFERENCIAS}

[Abujamra y Randall, 2019] Abujamra, R. y Randall, D. (2019). Chapter Five - Blockchain applications 
in healthcare and the opportunities and the advancements due to the new information technology framework. En S. Kim, G. C. Deka y Z. Peng (eds.), Advances in computers (pp. 14154). Ámsterdam: Países Bajos: https://doi. org/10.1016/bs.adcom.2018.12.002 个Ver página 119

[Ahram et al., 2017] Ahram, T., Sargolzaei, A., Sargolzaei, S. Daniels, J. y Amaba, B. (2017). Blockchain technology innovations (pp. 137-41). Santa Clara, EE. UU.: TEMSCON. DOI: https://doi.org/10.1109/TEMSCON . 2017.7998367 $\uparrow$ Ver página 114

[Alblooshi, 2018] Alblooshi, M., Salah, K. y Alhammadi, Y. (2018). Blockchain-based ownership management for medical IoT (MIoT) Devices. En International Conference on Innovations in Information Technology (IIT) (pp. 151-156). IEEE. DOI: https://doi.org/10.1109/ INNOVATIONS.2018.8606032 个Ver página

[Alwen, 2015] Alwen, J., Fuchsbauer, G., Gazi, P., Park, S. y Pietrzak, K. (2015). Spacecoin: a cryptocurrency based on proofs of space. IACR Cryptology EPrint Archive, 528, 1-26. Recuperado de https://eprint.iacr.org/2015/528 $\uparrow$ Ver página

[Antonopoulos, 2014] Antonopoulos, A. M. (2014). Mastering Bitcoin: unlocking digital cryptocurrencies. Sebastopol, EE. UU.: O'Reilly Media. $\uparrow$ Ver página 114

[Arévalo-Ascanio, Bayona-Trillos y Rico-Bautista, 2015] Arévalo-Ascanio, J. G., Bayona-Trillos, R. A. y Rico-Bautista, D. W. (2015). Implantación de un sistema de gestión de seguridad de información bajo la ISO 27001: Análisis del riesgo de la información. Tecnura, 19(46), 123-134. DOI: https://doi.org/10.14483/udistrital. jour.tecnura.2015.4.a10 Ver página 119

[Bach, Mihaljevic y Zagar, 2018] Bach, L. M., Mihaljevic, B. y Zagar, M. (2018). Comparative Analysis of Blockchain Consensus Algorithms (pp. 1545-1550). Opatija, Croacia: MIPRO. Recuperado de https://doi.org/10.23919/MIPRO. 2018.8400278 $\uparrow$ Ver página 116

[Back, 1997] Back, A. (1997). Hashcash. Recuperado de http://www.cypherspace.org/ hashcash/ $\uparrow$ Ver página

[Back, 2002] Back, A. (2002). Hashcash-A denial of service counter-measure. Recuperado de http://www. Hashcash.Org/Papers/ Hashcash.Pdf. $\uparrow$ Ver página

[Bitcoincash, 2017] Bitcoincash (2017). Bitcoin cash: peer-to-peer electronic cash. Recuperado de https: //www.Bitcoincash.org/ $\uparrow$ Ver página

[Bitmoney, 2019] Bitmoney Inc. (2019). Bitmoney: follow the phoenix. Recuperado de https:// bitmoney . ws / $\uparrow$ Ver página

[] BitShares Blockchain Foundation (2013). BitShares 2.0 - Industrial-Grade Decentralized (DPoS) eco-system on blockchain. Recuperado de https://bitshares.org/ $\uparrow$ Ver página

[] Block.one (2017). Eosio-blockchain software architecture. Recuperado de https://eos.io/ $\uparrow$ Ver página

[Bocek, Rodrigues, Strasser y Stiller, 2017]

Bocek, T., Rodrigues, B. B., Strasser, T. y Stiller, B. (2017). Blockchains everywhere-a use case of blockchains in the pharma supply-chain. En IFIP/IEEE Symposium on Integrated Network and Service Management (IM) (pp. 772-777). Lisboa, Portugal. DOI: https: //doi.org/10.23919/INM.2017.7987376 个Ver página 122

[Bowman, 2018] Bowman, M. y Gendal, R. (2018). Hyperledger Sawtooth-PoET. Recuperado de https: / / sawtooth.hyperledger.org/ docs/core/releases/1.0/architecture/ poet.html $\uparrow$ Ver página

[Bragazzi, 2013] Bragazzi, N. L. (2013). From P0 to P6 medicine, a model of highly participatory, narrative, interactive, and 'augmented' medicine: some considerations on Salvatore Iaconesi's clinical story. Patient Preference and Adherence, 
7, 353-359. DOI: https://doi.org/10.2147/ ppa.s38578 $\uparrow$ Ver página 117

[Bragazzi y Del Puente, 2013] Bragazzi, N. L. y Del Puente, G. (2013). Why P6 medicine needs clinical psychology and a trans-cultural approach. Health Psychology Research, 1(1), 5. DOI: https://doi. org/10.4081/hpr.2013.e5 个Ver página 117

[Brandon, 2020] Brandon, Ch. (2020). Cryptocurrency market capitalizations. https: //coinmarketcap.com/ Recuperado de $\uparrow$ Ver página 116

[Burstcoin, 2014] Burstcoin Inc. (2014). Burstcoin-The Linux of blockchain. Recuperado de https:// www.burst-coin.org/ $\uparrow$ Ver página

[Cardano Foundation, 2020] Cardano Foundation (2020). Cardano - Home of the Ada cryptocurrency and technological platform. Recuperado de https://www.cardano.org/ 个Ver página

[Celiz, 2018] Celiz, R. C., De la Cruz, Y. E. y Sánchez, D. M. (2018). Cloud model for purchase management in health sector of Peru based on IoT and blockchain. En IEEE 9 th Annual Information Technology, Electronics and Mobile Communication Conference (IEMCON) (pp. 328-334). Vancouver, Canadá: IEEE. DOI: https://doi.org/10. 1109/IEMCON.2018.8615063 个Ver página

[Cohen, 2019] Cohen, B. (2019). Chia Network-A New blockchain and smart transaction platform that is easier to use, more efficient, and secure. Recuperado de https://www.chia.net/ $\uparrow$ Ver página

[CounterParty Inc, 2019] CounterParty Inc. (2019). Counterparty extends bitcoin in new and powerful ways. Recuperado de https: / / counterparty. io/ $\uparrow$ Ver página

[Crosby, Ghosh, Murimi y Chin, 2012] Crosby, G. V., Ghosh, T., Murimi, R. y Chin, C. (2012). Wireless body area networks for healthcare: a survey. International Journal of Ad Hoc, Sensor \& Ubiquitous Computing, 3(3), 1-26. DOI: https: // doi.org/ 10.5121/i jasuc.2012.3301 $\uparrow$ Ver página 122
[Dai] Dai, W. (s. f.). B-money. Recuperado de http: //www. weidai.com/bmoney.txt $\uparrow$ Ver página

[Diffie, 1976] Diffie, W. y Hellman, M.E. (1976). New directions in cryptography. IEEE Transactions on Information Theory, IT-22(6), 644-654. $\uparrow$ Ver página

[Dubovitskaya et al., 2018] Dubovitskaya, A., Xu, Z., Ryu, S., Schumacher, M. y Wang, F. (2018). Secure and Trustable Electronic Medical Records Sharing Using Blockchain. En AMIA. Annual Symposium Proceedings (pp. 650-659). American Medical Informatics Association. $\uparrow$ Ver página 120

[Dwork, 1993] Dwork, C. y Naor, M. (1993). Pricing via processing, or, combatting junk mail, advances in cryptology. CRYPTO 92: Lecture Notes in Computer Science, 740, 139-147. $\uparrow$ Ver página

[Ekblaw, Azaria, Vieira y Lippman, 2016] Ekblaw, A., Azaria, A., Vieira, T. y Lippman, A. (2016). MedRec: using blockchain for medical data access and permission management. En 2nd International Conference on Open and Big Data (OBD) (pp. 25-30). IEEE. DOI: https://doi.org/10.1109/OBD.2016.11 $\uparrow$ Ver página 120

[Ethereum Foundation, 2015] Ethereum Foundation (2015). Ethereum project. Recuperado de https://www.Ethereum.org/ $\uparrow$ Ver página 121

[Feng et al., 2019] Feng, Q., Debiao, H., Sherali, Z., Muhammad, K. K. y Kumar, N. (2019). A survey on privacy protection in blockchain system. Journal of Network and Computer Applications, 126, 45-58. DOI: https://doi.org/10.1016/ j.jnca.2018.10.020 个Ver página 114

[Finney, 2004] Finney, H. (2004). RPOW Reusable Proofs of Work. Recuperado de https://cryptome.org/rpow.htm $\uparrow$ Ver página

[GemOS Inc, 2018] GemOS Inc. (2018). The blockchain operating system. Recuperado de https://enterprise.gem.co/health/ 个Ver página 120, 123 
[Gervais et al., 2016] Gervais, A., Ghassan, O. K., Wüst, K., Vasileios, G., Ritzdorf, H. y Srdjan, C. (2016). On the security and performance of proof of work blockchains (pp. 3-16). Nueva York, EE. UU.: Association for Computing Machinery. Recuperado de http://dl.acm.org/ citation. cfm?doid=2976749.2978341

$\uparrow$ Ver página 114

[Gramoli, 2020] Gramoli, V. (2020). From blockchain consensus back to byzantine consensus. Future Generation Computer Systems, 107, 760-69. DOI: https://doi.org/10.1016/j.future. $2017.09 .023 \uparrow$ Ver página 116

[Griggs et al., 2018] Griggs, K. N., Ossipova, O., Kohlios, C. P., Baccarini, A. N., Howson, E. A. y Hayajneh, T. (2018). Healthcare blockchain system using smart contracts for secure automated remote patient monitoring. Journal of Medical Systems, 42(7), 130-37. Recuperado de https://dl.acm.org/doi/10.1007/ s10916-018-0982-x $\uparrow$ Ver página 120

[Guardtime, 2019] Guardtime (2019). Guardtime: enterprise blockchain. Recuperado de https: //guardtime.com/ $\uparrow$ Ver página

[Hassan y Primavera, 2017] Hassan, S. y Primavera, De F. (2017). The expansion of algorithmic governance: from code is law to law is code. Field Actions Science Reports, 17(17), 88-90. Recuperado de http://journals.openedition.org/ factsreports/4518 $\uparrow$ Ver página 116

[Health Level Seven International, 2011] Health Level Seven International (2011). Fast healthcare interoperability resources. Recuperado de https://www.hl7.org/fhir/ $\uparrow$ Ver página 121

[HealthBank Cooperative, 2019] HealthBank Cooperative (2019). Health Bank. Recuperado de https://www. healthbank.coop/ Hölbl, M., Kompara, M., Kamišali, A. y Nemec, L. (2018). A systematic review of the use of blockchain in healthcare. Symmetry, 10(470), 1-22. DOI: https://doi.org/10.3390/sym10100470 个Ver página 120

[Hölbl, Kompara, Kamišali y Nemec, 2018] Hölbl, M., Kompara, M., Kamišali, A. y Nemec, L. (2018). A systematic review of the use of blockchain in healthcare. Symmetry, 10(470), 1-22. DOI: https://doi.org/10.3390/sym10100470 个Ver página 118

[Infiniti Research Limited, 2018] Infiniti Research Limited (2018). Global remote patient monitoring market 2018-2022. Recuperado de https://www.technavio.com/report/ global-remote-patient-monitoring-mal rket-analysis-share-2018 $\uparrow$ Ver página 121

[Kshetri, 2017] Kshetri, N. (2017). Can blockchain strengthen the internet of things? IT Professional, 19(4), 68-72. DOI: https://doi.org/10. 1109/MITP.2017.3051335 个Ver página 123

[KovanGithub, 2014] Kovan Github (2014). Kovan-stable Ethereum public testnet. Recuperado de https://github.com/kovan-testnet/ proposal $\uparrow$ Ver página

[Kuo, Kim y Ohno-machado, 2017] Kuo, T., Kim, H. y Ohno-machado, L. (2017). Review blockchain distributed ledger technologies for biomedical and health care applications. Journal of the American Medical Informatics Association, 24(6), 1211-1220. DOI: https://doi.org/10.1093/ jamia/ocx068 4 Ver página 119

[Litecoin Foundation, 2017] Litecoin Foundation (2017). Litecoin Foundation: designing the future of global payments. Recuperado de https://litecoin-foundation.org/ $\uparrow$ Ver página

[McGhin, Choo, Liu y He, 2019] McGhin, T., Choo, K. K. R., Liu, C. Z. y He, D. (2019). Blockchain in healthcare applications: research challenges and opportunities. Journal of Network and Computer Applications, 135, 62-75. DOI: https: //doi.org/10.1016/j.jnca.2019.02.027 个Ver página 119 
[Merkle, 1980] Merkle, R. C. (1980). Protocols for public key cryptosystems. En Proc. 1980 Symposium on Security and Privacy (pp. 122-133). IEEE Computer Society. $\uparrow$ Ver página

[Mettler, 2016] Mettler, M. (2016). Blockchain technology in healthcare: the revolution starts here. En IEEE 18th International Conference on e-Health Networking, Applications and Services (Healthcom) (pp. 1-3). Múnich, Alemania: IEEE. DOI: https://doi.org/10.1109/ HealthCom.2016.7749510 个Ver página 120

[Modum.io AG, 2017] Modum.io AG (2017). Data integrity for supply chain operations, powered by blockchain technology (Whitepaper). Recuperado de https://modum.io/sites/ default/files/documents/2018-05/ modum-whitepaper-v.-1.0.pdf $\uparrow$ Ver página 122,123

[Nakamoto, 2008] Nakamoto, S. (2008). Bitcoin: a peer-to-peer electronic cash system. Recuperado de https://Bitcoin.org/en/ $\uparrow$ Ver página 114, 116

[Nchinda, Cameron, Retzepi y Lippman, 2019] Nchinda, N., Cameron, A., Retzepi, K. y Lippman, A. (2019). MedRec: a network for personal information distribution. En International Conference on Computing,Networking and Communications (ICNC) (pp. 637-641). Honolulu, EE. UU.: IEEE. DOI: https: //doi.org/10.1109/ICCNC.2019.8685631 $\uparrow$ Ver página 120

[Neo Foundation, 2017] Neo Foundation (2017). $\mathrm{NEO}-A n$ open network for smart economy. Recuperado de https://neo.org/ $\uparrow$ Ver página

[Nivia y Jaramillo, 2018] Nivia, A. M. y Jaramillo, I. (2018). La industria de sensores en Colombia. Tecnura, 22(57), 44-54. DOI: https: / / doi . org/ $10.14483 / 22487638.13518 \uparrow$ Ver página 121

[Nix Platform, 2018] Nix Platform (2018). NIX Platform-total privacy for all. Recuperado de https://nixplatform.io/ $\uparrow$ Ver página
[Nomura Research Institute, 2016] Nomura

Research Institute (2016). Survey on blockchain technologies and related services. Recuperado de https://www.meti.go.jp/english/ press/2016/pdf/0531_01f.pdf $\quad \uparrow$ Ver página 114

[P4Titan, 2014] P4Titan (2014). Slimcoin. A peer-to-peer crypto-currency with proof-of-burn 'Mining without powerful hardware'. Recuperado de http://www.slimcoin.org $\uparrow$ Ver página

[Peterson, Deeduvanu, Kanjamala y Boles, 2016] Peterson, K., Deeduvanu, R., Kanjamala, P. y Boles, K. (2016). A blockchain-Based Approach to Health Information Exchange Networks (pp. 1-10). NIST Workshop Blockchain Healthcare. $\uparrow$ Ver página 120

[Pirtle, 2018] Pirtle, C. y Ehrenfeld, J. (2018). Blockchain for Healthcare: The Next Generation of Medical Records? Journal of Medical Systems, 42(9), 1-3. DOI: https: //doi.org/10.1007/s10916-018-1025-3 个Ver página 119

[PIVX Community, 2018] PIVX Community (2018). PIVX: private-instant-verified-transaction (Tx) (whitepaper). Recuperado de https: //pivx.org/white-papers-2/ $\uparrow$ Ver página

[po.et Foundation, 2018] po.et Foundation (2018). The decentralized protocol for content ownership, discovery and monetization of media. Recuperado de https://research.binance.com/en/ projects/poet $\uparrow$ Ver página

[Popov, 2018] Popov, S. (2018). The Tangle (Whitepaper). Recuperado de https://www. IoTa.org/foundation/research-papers $\uparrow$ Ver página

[Rabin, 1978] Rabin, M. O (1978). Digitalized signatures. En R. A. DeMillo et al. (eds.), Foundations of secure computation (pp. 155-168). Cambridge, EE. UU.: Academic Press. $\uparrow$ Ver página 
[Ruge y Pérez, 2017] Ruge, I. A. y Pérez, W. J. (2017). Diagnóstico tecnológico del uso de dispositivos programables en la industria boyacense. Caso de estudio: cadena agroindustrial de la panela. Tecnura, 21(52), 130-147. DOI: https://doi.org/10.14483/udistrital. jour.tecnura.2017.2.a09 Ver página 121

[Sajana, 2018] Sajana, P., Sindhu, M. y Sethumadhavan, M. (2018). On blockchain applications: Hyperledger Fabric and ethereum. International Journal of Pure and Applied Mathematics, 118(18), 2965-2970. $\uparrow$ Ver página

[Sankar, Sindhu y Sethumadhavan, 2017] Sankar, L. S., Sindhu, M. y Sethumadhavan, M. (2017). Survey of consensus protocols on blockchain applications (pp. 1-5). Coimbatore, Tamil Nadu, India: IEEE. DOI: https://doi.org/10. 1109/ICACCS.2017.8014672 个Ver página 116, 122

[Schwartz, 2018] Schwartz, D., Youngs, N. y Britto, A. (2018). The Ripple Protocol Consensus Algorithm (Whitepaper). Recuperado de https://ripple.com/files/ripple_ consensus_whitepaper.pdf $\uparrow$ Ver página

[Sedrati, Abdelraheem y Raza, 2018] Sedrati,

A., Abdelraheem, M. A. y Raza, S. (2018). Blockchain and IoT: mind the gap. Interoperability, Safety and Security in IoT, 2, 113-122. Springer International Publishing. DOI: https://doi. org/10.1007/978-3-319-93797-7_13 个Ver página 123

[Seeley, 2018] Seeley, L. (2018). Hyperledger Sawtooth-Pbft. Recuperado https://github. com/hyperledger/sawtooth-rfcs/blob/ master/text/0019-pbft-consensus.md $\uparrow$ Ver página

[Shae, 2017] Shae, Z. y Jeffrey, J. P. P. (2017). On the Design of a Blockchain Platform for Clinical Trial and Precision Medicine. En IEEE 37th International Conference on Distributed Computing Systems (ICDCS) (pp. 1972-1980). DOI: https:
//doi.org/10.1109/ICDCS.2017.61 个Ver página 123

[Shrobe, Shrier y Pentland, 2018] Shrobe, H., Shrier, D. y Pentland, A. (2018). Chapter 15. Enigma: decentralized computation platform with guaranteed privacy. En New Solutions for Cybersecurity (pp. 425-414). Cambridge, EE. UU.: MIT Press. $\uparrow$ Ver página 123

[Silent Notary, 2018] Silent Notary (2018). Decentralized notary system. Recuperado de https://silentnotary.com/ $\uparrow$ Ver página

[Snow, 2018] Snow, P., Deery, B., Lu, J., Johnston, D. y Kirby, P. (2018). Factom. Business processes secured by immutable audit trails on the blockchain. Recuperado de https://www.factom.com/ $\uparrow$ Ver página

[Steem Inc, 2017] Steem Inc. (2017). Steem. An incentivized, blockchain-based, public content platform (whitepaper). Recuperado de https: //steem.io/steem-whitepaper.pdf $\uparrow$ Ver página

[Stornetta, 1991] Stornetta, W. S. y Haber, S. (1991). How to time-stamp a digital document. Journal of Cryptology, 3(2), 99-111. $\uparrow$ Ver página

[Szabo, 1994] Szabo, N. (1994). Smart contracts. Recuperado de http://www . fon.hum.uva.nl/rob/Courses/ InformationInSpeech/CDROM/ Literature/LOTwinterschool2006/ szabo.best. vwh. net/smart. contracts . html. $\uparrow$ Ver página

[Szabo, 1996] Szabo, N. (1996). Smart contracts: building blocks for digital markets. EXTROPY: The Journal of Transhumanist Thought, (16). $\uparrow$ Ver página

[Szabo, 2005] Szabo, N. (2005). Bit Gold. Recuperado de https://nakamotoinstitute.org/ bit-gold/ 个Ver página

[Talukder, Chaitanya, Arnold y Sakurai, 2018] Talukder, A. K., Chaitanya, M., Arnold, D. y Sakurai, K. (2018). Proof of disease: a blockchain 
consensus protocol for accurate medical decisions and reducing the disease burden. En IEEE SmartWorld, Ubiquitous Intelligence Computing, Advanced Trusted Computing, Scalable Computing Communications, Cloud Big Data Computing, Internet of People and Smart City Innovation (SmartWorld/SCALCOM/UIC/ATC/CBDCom/IOP/SCI) (pp. 257-262). IEEE. DOI: https://doi.org/ 10.1109/SmartWorld.2018.00079 $\uparrow$ Ver página 120

[The Linux Foundation, 2017] The Linux Foundation. (2017). Hyperledger Fabric. Recuperado de https://www.hyperledger. org/projects/fabric $\uparrow$ Ver página 123

[The NEM Group Ltd, 2018] The NEM Group Ltd. (2018). NEM techical reference. Recuperado de https://nemplatform.com/wp-content/ uploads/2020/05/NEM_techRef.pdf $\uparrow$ Ver página

[Trew, 2017] Trew, Ch., Brandon, G. y Dorier, N. (2017). Stratis blockchain solutions (whitepaper). Recuperado de https://stratisplatform. com/files/Stratis_Whitepaper.pdf $\uparrow$ Ver página

[Vasin, 2014] Vasin, P. (2014). BlackCoin's Proof-of-Stake Protocol V2. Recuperado de https://blackcoin.co/ blackcoin-pos-protocol-v2-whitepaper. pdf $\uparrow$ Ver página

[Vauplane, 2017] Vauplane, H. (2017). Blockchain: la question de la preuve par consensus au coeur de la gouvernance. Le Mois En Revue, 796, 16-18. Recuperado de http://www.revue-banque.fr/ risques-reglementations/chronique/ Blockchain-question-preuve-par-consen\ sus-au-coeur $\uparrow$ Ver página 116

[Waves Technologies, 2016] Waves Technologies (2016). Open blockchain platform and development toolset for Web 3.0 applications and decentralized solutions. Recuperado de https://wavesplatform.com/ $\uparrow$ Ver página

[Williams-Grut, 2016] Williams-Grut,

O. (2016). Estonia is using the technology behind bitcoin to secure 1 million health records. Business Insider. Recuperado de https: / / www. businessinsider.com/ guardtime-estonian-health-records-il ndustrial-Blockchain-Bitcoin-2016-3 个Ver página 120

[World Health Organization, 2005] World Health Organization (WHO) (2005). Constitution of the World Health Organization. Recuperado de https://apps.who.int/gb/bd/PDF/ bd47/EN/constitution-en.pdf?ua=1 $\uparrow$ Ver página 117

[World Health Organization, 2010] World Health Organization (WHO) (2010). Growing threat from counterfeit medicines. Bulletin of the World Health Organization, 88(4), 247-48. DOI: https: //doi.org/10.2471/blt.10.020410 个Ver página 122

[World Health Organization, 2018] World Health Organization (WHO) (2018). Substandard and falsified medical products. Recuperado de https://www.who.int/ news-room/fact-sheets/detail/ substandard-and-falsified-medical-\ products $\uparrow$ Ver página 122

[Zhang, Xue y Huang, 2016] Zhang, J., Xue, N. y Huang, X. (2016). A secure system for pervasive social. IEEE Access, 4, 9239-9250. DOI: https : // doi.org/10.1109/ACCESS.2016.2645904 个Ver página 123

[Zheng et al., 2017] Zheng, Z., Xie, S., Dai, H., Chen, X. y Wang, H. (2017). An overview of blockchain technology: architecture, consensus, and future trends (pp. 557-564). DOI: https://doi.org/ 10.1109/BigDataCongress.2017.85 $\uparrow$ Ver página 116 